\title{
Theoretical Research on the Stability Analysis and De-noising Methodologies for Single-chip Microcomputer Control System
}

\author{
Zhuangzhuang Liu \\ School of Information and Electrical Engineering, China University of Mining and Technology, \\ Xuzhou 221116, China \\ 137487513@qq.com
}

Keywords: Stability Analysis; Single-chip Microcomputer; Control System; Evaluation Method.

\begin{abstract}
In this paper, we conduct research on the stability analysis and de-noising methodologies for single-chip microcomputer control system. As the sensor technology, microelectronics technology and the continuous development of single chip microcomputer technology for the perfection of the intelligent temperature measurement and control system and control function is aiming at improving measurement precision and anti-interference ability enhancement. In the experiment part, the numerical simulation indicates the feasibility of our approach.
\end{abstract}

\section{Introduction}

With the progress of science and technology and the rapid development of economy, the intelligent home furnishing, and Internet of things technology have taken a rapid development and progress. In recent, more and more researchers have set their interest on the topic of stability analysis and de-noising methodologies for single-chip microcomputer control system. Temperature measurement and control has wide application in industrial field. As the sensor technology, microelectronics technology and the continuous development of single chip microcomputer technology for the perfection of the intelligent temperature measurement and control system and control function is aiming at improving measurement precision and anti-interference ability enhancement. Because single chip has high integration, strong function, small volume, low price, the advantages of anti-interference ability is better than that of general CPU. As a result, higher requirements on control precision and low cost of industrial measurement and control system often as a digital controller to replace analog controller by single chip microcomputer. Due to the PID control technology is mature and control structure is simple which is easy to adjust parameters and need not the digital model of controlled object can be adjust [1-2]. We usually adopts PID algorithm in the temperature measurement and control system. However, due to the characteristics of the controlled object is different, the parameters of the PID algorithm is often different. The goal and function of the ITS include the following aspects: to improve the safety level of traffic; congestion; keep the traffic smooth; improve the transport network capacity; to help people in the use of more safe, convenient traffic, fast, comfortable; reduce the pollution degree to the environment of transportation and energy saving; improve the transportation efficiency of production and economic benefit. To this end, this paper introduces a kind of based on single chip microcomputer temperature control device. In its internal default set of PID parameters which are depended on the controlled object automatically choose the suitable PID parameters to control the temperature. In addition to the current model, the staff can also according to the specific requirements for a given temperature control and to modify each PID parameters online. Temperature measurement and control system is a closed-loop control system with the temperature of the temperature sensor will detect the A/D converted into computer. Compared with the set value deviation, the deviation correction according to the PID algorithm and the corresponding control actuators is to adjust the controlled object so as to realize the temperature control [3-4].

In the working process of the system, a temperature detection and conversion circuit converts the temperature of the measured object into the voltage signal and the voltage signal is converted to a digital signal by $\mathrm{A} / \mathrm{D}$ converter after into single chip microcomputer. At the same time, the 
corresponding to a given temperature control of multiple sets of comparison to find a temperature range and preloading PID parameters to output the corresponding initial temperature. Initial value corresponding to the temperature of the controlled object such as motor speed initial value by D/A converter converted into analog voltage control continuous adjustable controller to effectively drive electromagnetic governor controlled object and the speed of the object after the conversion circuit and A/D converter feedback to the single-chip microcomputer. Traditional way of temperature control for conventional relay drive contactor control, it can only be done within a certain range of temperature control, and belongs to the contact control, therefore, the low control precision, the working poor reliability, large energy consumption, the cost of higher shortcomings. Compared with the output speed of initial value, its deviation by PID program could be calculated the output again after within the prescribed period of time cycle until reach in the range of allowable error equal speed and output speed value of the initial value. In addition, de-noising term acts as a significant role in the process of enhancing the stability of the system. To deal with the stressed issues, we did research on the stability analysis and de-noising methodologies for single-chip microcomputer control system in this paper.

\section{The Proposed Methodology}

The Principles Signal De-noising. The reliability of the power transformer mainly depends on the insulation condition, and one of the main causes of transformer insulation aging and damage is PD, so transformer PD on-line monitoring has high theoretical and practical value to improve power system reliability and economy. Wavelet transform is a time-frequency multi-resolution analysis method, and it is widely used in signal de-noising and fault diagnosis. Control the number of input pulse stepper motor, frequency and the motor on the order of each phase winding can be running characteristic of various kinds of needs. Especially with digital equipment, embodies the superiority of bigger and therefore it is widely used in digital control system. When using a timer interrupt mode control motor speed, in fact, is constantly changing the size of the timer load value. In the following formulas we introduce the basic and important steps of the transform. For each mode, compute the analytic signal by means of the Hilbert transform in order to obtain a unilateral frequency spectrum.

$$
\left(\delta(t)+\frac{j}{\pi t}\right) \times u_{k}(t):\left[\left(\delta(t)+\frac{j}{\pi t}\right)\right] e^{-j w_{k} t}
$$

In the whole process mentioned above, the decomposition level plays an important role in wavelet de-noising. Under excessive decomposition levels, the signal information will be loss, the SNR will be decreased and the computational complexity is increased. On the other side, the noise reduction effect is not ideal, and with limited SNR which is shown in the formula 2.

$$
W_{k}=\operatorname{span}\left(2^{j / 2} \Psi_{i}\left(2^{j} t-k\right): 1 \leq i \leq r, k \in Z\right)
$$

Signals are decomposed into a series of components called coefficients ranging from the highest to the lowest frequencies scales. Such decomposition is performed based on convolution between original signal and the selected mother wavelets, which shifts along the time scale using translation and scale factors. The following equations illustrate the corresponding transform.

$$
\begin{aligned}
& R(t)=T_{c}(t) \cos w_{c} t+T_{s}(t) \sin w_{c} t \\
& T_{c}(t)=\frac{2}{\sqrt{M}} \sum_{n=1}^{M} \cos \left(\Psi_{n}\right) \cos \left(w_{m} t \cos \alpha_{n}+\varphi\right) \\
& T_{s}(t)=\frac{2}{\sqrt{M}} \sum_{n=1}^{M} \sin \left(\Psi_{n}\right) \cos \left(w_{m} t \cos \alpha_{n}+\varphi\right)
\end{aligned}
$$


Basically, better de-noising performance can be achieved if the selected mother wavelets have high correlation with real signals. The effect will be tested in the simulation section.

The Hardware Description. Using photoelectric isolating circuit composed of optical isolator isolates controller and the external drive circuit which makes the change of the external circuit doesn't affect or damage to the control system so as to improve the reliability of the system and strengthen thousands of anti-disturbance ability. Optical isolator connection attention signal positive and negative logic. Optical isolator of the input and output ground wire must be separated from each other, and the input and output power must be separate two power supply. As we will use the same power supply, external disturbance signals may be through the power to the system. Time delay method is called after each reversing a delay subroutine, to delay again after the commutation, so the cycle frequency of CP pulses could be sent, or reversing cycle. Delay subroutine of delay time and the reversing process time and is used in the CP pulse cycle. This method is simple, less resource-intensive, all by software to realize, call different subroutines can realize the operation of the different speed; But the CPU time is long, can't handle other work at runtime, so only suitable for simple control process. The two-way phase sequence waveform excitation is shown below.

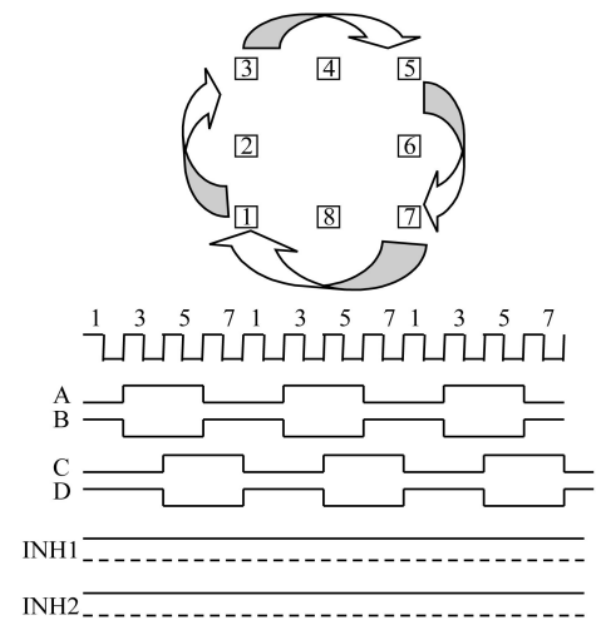

Fig. 1 The Two-way Phase Sequence Waveform Excitation for the System

The core of the intelligent temperature control system adopted the MSP430 series microcontroller, and through the data acquisition circuit, the temperature field in the oven equipment remote control and detection, and can according to the preset temperature threshold, automatic heating or cooling operation. The user can through the data display circuit, temperature field check equipment, and can through the keyboard in the field of intelligent temperature controller Settings. At the same time, the user can also in remote by RS-485 bus through the network through the serial port circuit of controller, remote temperature settings and data access to it. It made great engineers in the control system of the complex distributed installation and commissioning work. The temperature control system which can be in accordance with the requirements for field devices and practical need to set the control needs to go through several stages to complete will set each step required to keep the temperature field of value and need to observe the relevant information. This system is real-time gathering data and at the same time of acquisition for the test comparison system may at any time according to the change of temperature and make the corresponding adjustment to control the heating system. In the figure 2 we show the structure of the system in detail. 


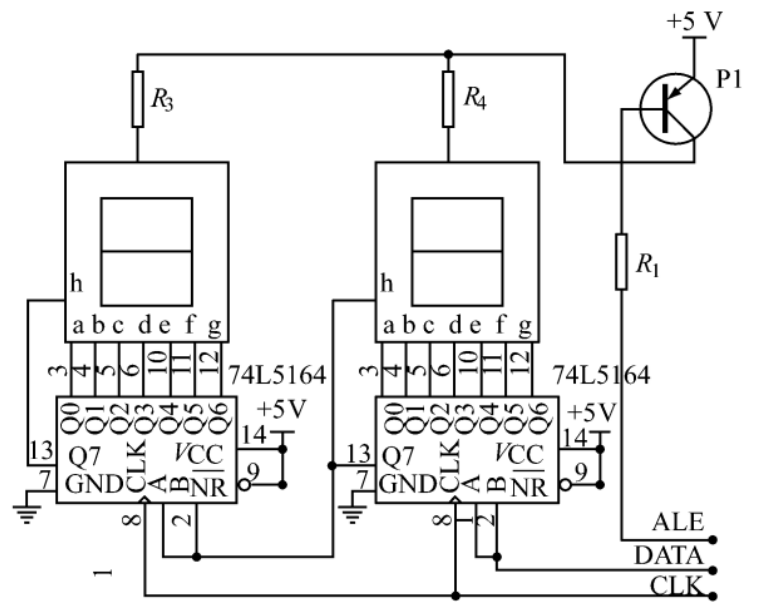

Fig. 2 The Structure of the Proposed System

Optical isolator connection attention signal positive and negative logic. Optical isolator of the input and output ground wire must be separated from each other, and the input and output power must be separate two power supply. Control algorithm is the core part of the control system which control system's stability and control quality is the key to good. This system is a closed-loop control computer control system directly and it works in accordance with a certain amount of sampling period $\mathrm{T}$ to be accused of samples and through the control algorithm to calculate the control quantity in order to control as well as the output control actuators. The next section will show the simulation.

\section{Experiment and Simulation}

In this section, we conduct numerical simulation for our proposed system and analyze the effect of the de-noising operation. In the testing system, temperature sampling interrupt handlers by temperature sampling, digital filtering and the temperature display program. In order to verify the feasibility of the inverter design, reduce design flaws caused by subsequent losses, through the MATLAB Simulink simulation capabilities can be easily and intuitively get results.

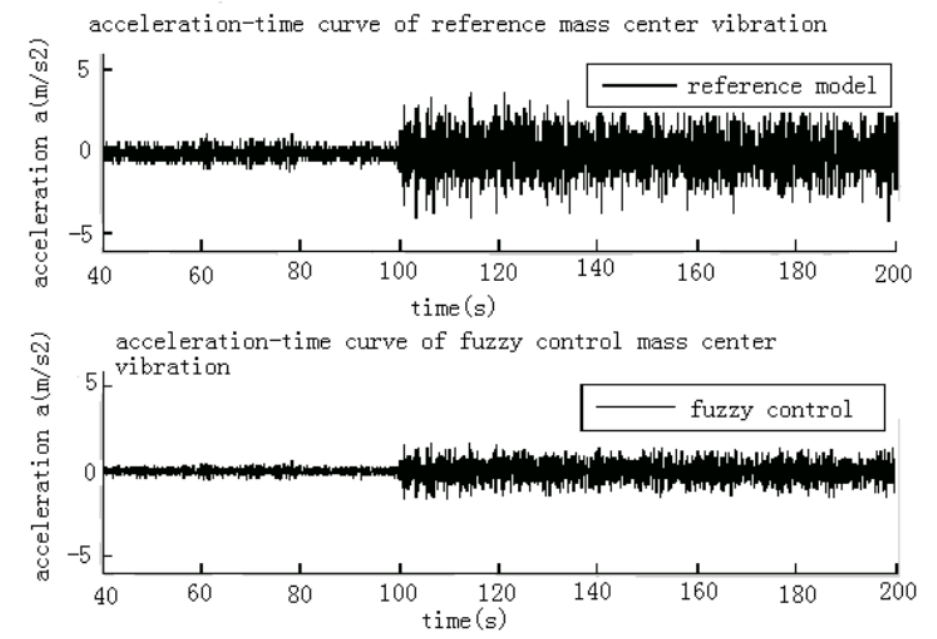

Fig. 3 The Simulation Result for Our Proposed System

\section{Conclusion and Summary}

In recent, more and more researchers have set their interest on the topic of stability analysis and de-noising methodologies for single-chip microcomputer control system. Temperature measurement and control has wide application in industrial field. In this paper, we conduct research on the stability analysis and de-noising methodologies for single-chip microcomputer control system. Through analyzing the hardware design and software development, combined with the de-noising steps. Our 
method is finalized with numerical analysis and simulation. In the near future, we plan to conduct more related research to level-up the overall performance of the system.

\section{References}

[1] Yizhuang S. Based on Single-chip Microcomputer Temperature Control System Design and Realization-PC Design[J]. Electrical Engineering, 2014.

[2] Yuan-shuo F, Ji-jiang S. The design of temperature and humidity measurement and control system based on single-chip microcomputer[J]. Journal of Shandong University of Technology, 2014.

[3] Juan L, Lihua D. Study on Capillary Vessel Dehumidification System Based on Single-chip Microcomputer Control[J]. Marine Electric \& Electronic Engineering, 2014.

[4] Dong-hui W, Xia Y. Design of Underground Water Curtain Automatic Dust Control System Based on AT89S52 Single-chip Microcomputer[J]. Coal Mine Machinery, 2014. 